David H. Roberts, 1 Robert I. Potash, 1 John F. C. Wardle, 1 Alan E. E. Rogers, 2 and Bernard F. Burke ${ }^{3}$

'Brandeis University, ${ }^{2}$ Haystack Observatory, ${ }^{3}$ M. I. T.

Linear polarization measurements at milliarcsecond resolution have been made at $\lambda 6 \mathrm{~cm}$ using four stations of the U. S. VLBI Network and the Mark III recording system. Galibration of the cross-polarization contamination of the feeds was done using the cross- to parallel-hand fringe ratios of the essentially unpolarized source $0 Q 208$, and is believed accurate to $0.5 \%$. Other sources, such as AO $0235+164$ and 0106+013, which are nearly unresolved in the unpolarized fringes, show clear signs of resolution in their polarized fringes. A preliminary map of the BL Lacertae object OJ 287 is compatible with a weakly-polarized optically-thick core and a highly-polarized optically-thin jet about 5 mas in length.

\title{
OBSERVATIONS
}

Data were taken for 24 hours in December 1981 with the 120-foot Haystack, 140-foot Green Bank, and 130-foot Owens Valley antennas, and the phased-up 27 antennas of the Very Large Array, using the Mark III recording system. Seven 2-MHz tracks each of right and left circularlypolarized radiation ( $R C P$ and LCP), centered at a sky frequency of 4983 $\mathrm{MHz}(\lambda 6 \mathrm{~cm})$, were recorded at Green Bank and the VLA, while only LCP was available at Haystack and Owens Valley. All possible parallel- and crosshand correlations were performed on the Haystack Mark III correlator.

\section{POLARIZATION CALIBRATION}

Detection of the RCP and LCP components of the source radiation (the electric fields $E_{R}$ and $E_{L}$ ) in each of the two elements of an interferometer enables one to measure the Stokes parameters ( $I, Q, U, V)$, where $I$ is the total intensity, $Q$ and $U$ are the linearly-polarized components, and $V$ is the circularly-polarized component. These are related to the complex linear polarization $P$ by $P=Q+i U=m I e^{2 i} \chi$, where $m$ is the fractional linear polarization and $X$ is the linear polarization position angle. Any real antenna and feed system will have arbitrary gain and some crosspolarization response, and the voltage induced in given right- and left-

+ Discussion on page 420

R. Fanti et al. (eds.), VLBI and Compact Radio Sources, 35-38.

(C) 1984 by the IAU. 
circularly polarized feeds may be written approximately as

$$
v_{R}=G_{R}\left(E_{R} e^{-i \phi_{p}}+D_{R} E_{L} e^{+i \phi_{p}}\right), \quad V_{L}=G_{L}\left(E_{L} e^{+i \phi_{p}}+D_{L} E_{R} e^{-i \phi_{p}}\right),
$$

where the G's and D's are complex gains and cross-talks, and $\phi_{p}$ is the parallactic angle. If all cross-correlations of the voltages are formed, and terms second-order in $m$ and $D$ are dropped, the results are

$$
\begin{aligned}
L_{1} L_{2}^{*} & =e^{i\left(\psi_{1}-\psi_{2}\right)} G_{1 L} G_{2 L}^{*}(I-V) e^{i\left(+\phi_{1}-\phi_{2}\right)}, \\
R_{1} L_{2}^{*} & =e^{i\left(\psi_{1}-\psi_{2}\right)} G_{1 R} G_{2 L}^{*}\left[\left(m I e^{+2 i x}\right) e^{i\left(-\phi_{1}-\phi_{2}\right)}\right. \\
& \left.+D_{2 L}^{*}(I+V) e^{i\left(-\phi_{1}+\phi_{2}\right)}+D_{1 R}(I-V) e^{i\left(+\phi_{1}-\phi_{2}\right)}\right],
\end{aligned}
$$

plus similar equations for RR and LR. Here $\psi_{1}$ and $\psi_{2}$ are time-variable phases due to the differences in clock rate, atmosphere, etc., at the two stations. In order to calibrate the polarization response, it is necessary to put successive cross-hand fringes in the same phase-frame. Since the parallel-hand fringes on a given baseline at a given epoch are contaminated by exactly the same phases $\psi_{1}$ and $\psi_{2}$ as are the crosshand fringes, their ratio is free of this phase ambiguity. Thus we form cross- to parallel-hand fringe ratios such as

$$
\frac{\mathrm{R}_{1} \mathrm{~L}_{2}^{*}}{\mathrm{~L}_{1} \mathrm{~L}_{2}^{*}}=\frac{\mathrm{G}_{1 \mathrm{R}}}{\mathrm{G}_{1 \mathrm{~L}}}\left[\mathrm{~m} \mathrm{e}^{2 \mathrm{i}\left(\mathrm{x}-\phi_{1}\right)}+\mathrm{D}_{2 \mathrm{~L}}^{*} \mathrm{e}^{2 i\left(-\phi_{1}+\phi_{2}\right)}+\mathrm{D}_{1 \mathrm{R}}\right] \text {, }
$$

neglecting terms of order V/I since the circular polarization of almost all compact radio sources is known to be small. These relations still contain the unknown (and possibly time-variable) ratios of right and left instrumental gains at a given stations, e.g., $\left(G_{1 R} / G_{1 L}\right)$. Fortunately, the ratio of parallel-hand fringes (RR/LL) on the same baseline involves the same gain ratios, and may be used to determine how they change in time. Due to the method of phasing-up the array, there was substantial R-L phase drift for the VLA, but essentially no drift at Green Bank. The amplitude part of the $\mathrm{R}-\mathrm{L}$ gain ratio proved to be quite stable (better than $1 \%$ ). With this information, the cross- to parallelhand fringe ratios contain one fixed vector and one or two rotating vectors, depending on the stations involved. Knowing the parallactic angles at each station for each scan, it is straightforward to solve for $m$ and the D's. The single overall phase constant was determined by comparing the VLBI results with those obtained simultaneously at arcsecond resolution for sources which are substantially unresolved by VLBI.

Two independent sets of polarization calibration software were used to analyze the data. One is correlator-based, and determines the antenna cross-gains baseline-by-baseline; these routines use only the cross- to parallel-hand fringe ratios. The second set of routines does a full antenna-based solution, and is quite similar to that used to calibrate the VLA. Each set was applied to $0 Q 208=1404+286$, which 
TABLE 1: INSTRUMENTAL CALIBRATION

\begin{tabular}{|c|c|c|c|c|}
\hline \multirow[b]{2}{*}{ Station } & \multicolumn{2}{|c|}{ Correlator-Based } & \multicolumn{2}{|c|}{ Antenna-Based } \\
\hline & $\overline{D_{R}^{*}(\%, \text { phase })}$ & $\overline{D_{L}(\%, \text { phase })}$ & $\overline{D_{R}^{*}(\%, \text { phase })}$ & $D_{L}(\%$, phase $)$ \\
\hline G & $5.8(-160)$ & 11.0( & $6.2(-163)$ & 10.4( \\
\hline $\mathrm{Y}$ & $2.0(-135)$ & $1.4(-39)$ & $2.0(-134)$ & $1.2(-36)$ \\
\hline $\mathrm{K}$ & -- & $2.5(+139)$ & -- & $2.7(+142)$ \\
\hline 0 & -- & $4.1(+93)$ & -- & $4.2(+91)$ \\
\hline
\end{tabular}

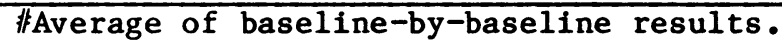

is essentially unpolarized $(<0.3 \%)$ at arcsecond resolution, yielding the cross-gains given in Table 1. The root-mean-square deviation of the fits to a individual correlators is typically about $0.4 \%$; we estimate that the overall polarization calibration is good to $0.5 \%$. There are small but measureable correlator-based differences in the cross-talks, probably due to non-identical band passes. For example, the term DYR has a value $1.8 \%$ when determined on the GY base-line, but $2.3 \%$ when determined on the KY baseline. Using the correlator-based calibration of the VLBI data, and only the short baselines KG and $O Y$, we find the source parameters given in Table 2. Although they are nearly unresolved in total intensity, the sources $0106+013,0235+164$, and $1749+049$ showed clear signs of resolution in their cross-hand fringes, indicating that their diffuse structure is more highly polarized than their compact components.

TABLE 2: SOURCE POLARIZATION

\begin{tabular}{|c|c|c|c|}
\hline \multirow[b]{2}{*}{ Source } & VLBI & \multicolumn{2}{|c|}{ VLA } \\
\hline & $\mathrm{m}(\%) \quad \mathrm{X}$ & $m(\%)$ & $x$ \\
\hline $\begin{array}{l}0106+013^{\text {非 }} \\
0235+164^{\text {非 }}\end{array}$ & $\begin{array}{ll}3.4 & -46 \\
1.3 & +38\end{array}$ & $\begin{array}{l}3.5 \\
1.8\end{array}$ & $\begin{array}{l}-52 \\
+44\end{array}$ \\
\hline OQ 208 & $<0.3$ & 非非 & 非 \\
\hline $1749+049^{\# \mid}$ & 4.5 & 非非 & 非 \\
\hline
\end{tabular}

POLARIZATION STRUCTURE OF OJ 287

A preliminary map of the polarized flux of OJ $287=0851+202$ was produced by the complex-polarization method. The cross- to parallel-hand fringe ratios were calibrated using the correlator-by-correlator crosstalks determined from $O Q 208$; the result of projecting the ratios onto the u-axis is shown in Figure 1. These cross- to parallel-hand fringe ratios were converted into polarization visibilities $P(u, v)$ using a model $I_{m}(x, y)$ of the total intensity distribution of this nearly-unresolved source, by multiplication with the corresponding visibility $I_{m}(u, v)$. A model consisting of a point source plus a 5 mas gaussian with flux ratio $5: 1$ and 5 mas separation was adopted, but the results are almost the same if a single point source is used instead. After a $u-v$ taper corresponding to angular resolution of 3 mas, a one-dimensional Fourier transform and a 


\section{RAW POLARIZATION VISIBILITY}
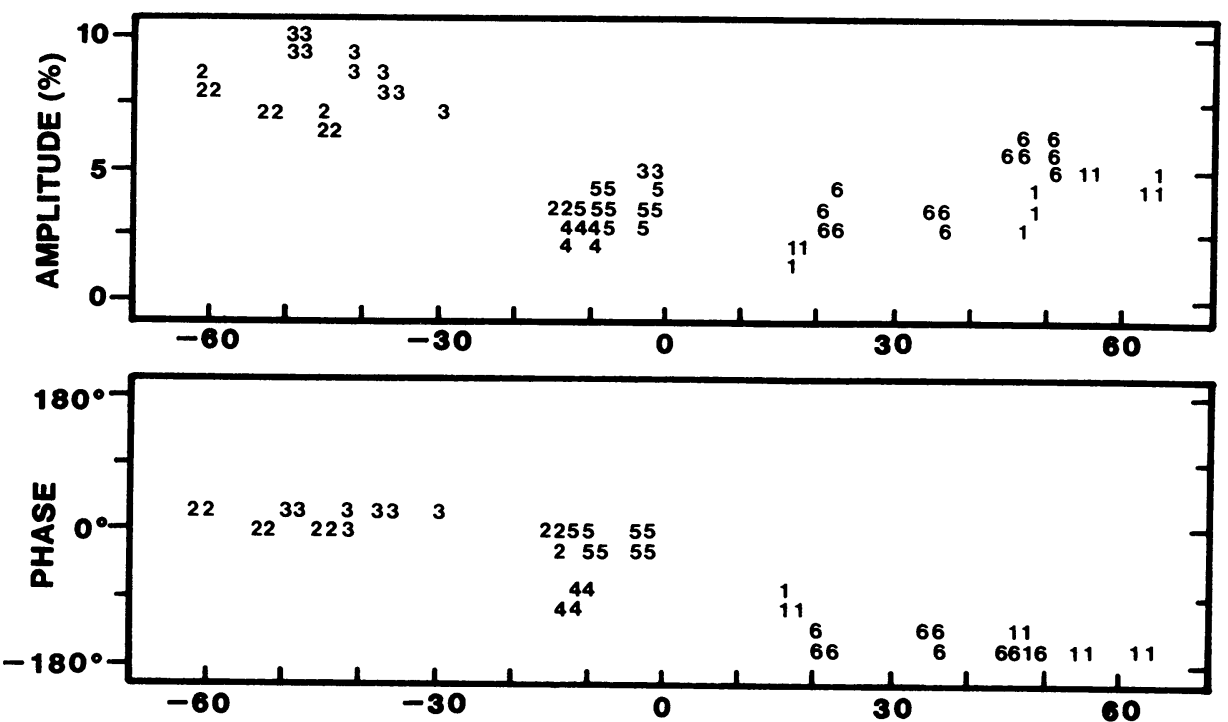

PROJECTED UV DISTANCE (MILLIONS OF WAVELENGTHS)

Figure 1. Cross- to parallel-hand fringe ratios for 0J 287, projected onto the u-axis. The various symbols distinguish the six correlators: $1=\mathrm{RL}(\mathrm{GY}), 2=\mathrm{LR}(\mathrm{GY}), 3=\mathrm{LR}(\mathrm{KY}), 4=\mathrm{LR}(\mathrm{OY}), 5=\mathrm{LR}(\mathrm{KG}), 6=\mathrm{LR}(\mathrm{OG})$.

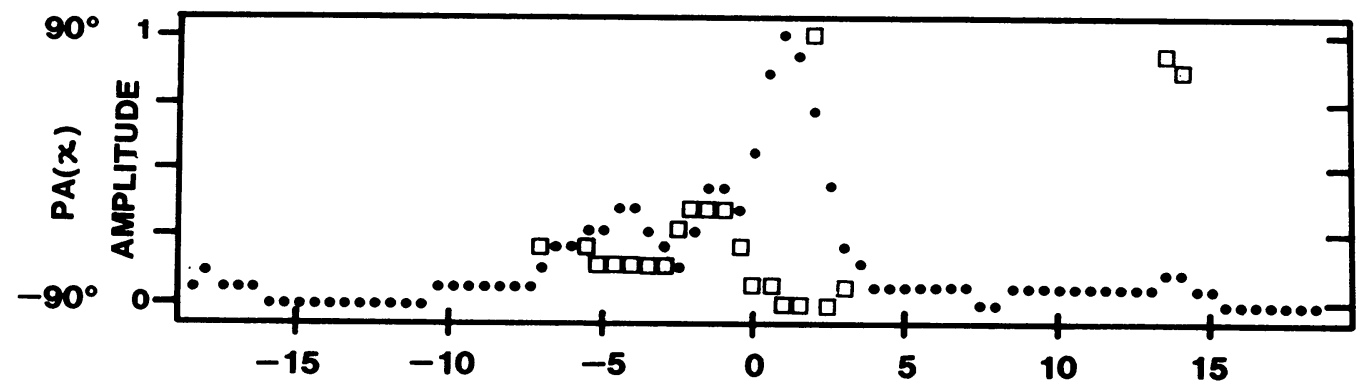

\section{POSITION (MILLIARCSEC)}

Figure 2. Complex polarization of 0J287, along $\mathrm{PA}=90^{\circ}$. The dots and squares are amplitude and position angle; the amplitude scale is arbitrary.

complex clean led to the map in Figure 2. The map is consistent with a weakly-polarized core and a highly-polarized jet, with significant changes in polarization position angle that may be related to changes in optical depth and/or orientation of magnetic field across the source.

The NRAO is operated by Associated Universities, Incorporated, under contract with the NSF. This research was supported in part by the NSF. 